\title{
ALMA long baseline phase calibration using phase referencing
}

\author{
Yoshiharu Asakia ${ }^{\mathrm{a}, \mathrm{b}}$, Satoki Matsushita ${ }^{\mathrm{c}}$, Edward B. Fomalont ${ }^{\mathrm{d}, \mathrm{b}}$, Stuartt A. Corder ${ }^{\mathrm{b}}$, Lars-Ake \\ Nyman $^{\mathrm{b}}$, William R. F. Dent ${ }^{\mathrm{b}}$, Neil M. Philips ${ }^{\mathrm{b}}$, Akihiro Hirota ${ }^{\mathrm{a}, \mathrm{b}}$, Satoko Takahashi ${ }^{\mathrm{a}, \mathrm{b}}$, \\ Baltasar Vila-Vilaro ${ }^{\mathrm{b}}$, Bojan Nikolic ${ }^{\mathrm{e}}$, Todd R. Hunter ${ }^{\mathrm{d}}$, Anthony Remijian ${ }^{\mathrm{d}}$, and Catherine \\ Vlahakis ${ }^{\mathrm{d}}$ \\ ${ }^{a}$ National Astronomical Observatory of Japan, Joaquin Montero 3000 Oficina 702, Vitacura, \\ Santiago, 763 0409, Chile \\ bJoint ALMA Observatory, Alonso de Córdova 3107, Vitacura, Santiago, 763 0355, Chile \\ ${ }^{c}$ Academia Sinica Institute of Astronomy and Astrophysics, P.O. Box 23-141. Taipei 10617, \\ Taiwan R.O.C. \\ ${ }^{d}$ National Radio Astronomy Observatory, Edgemont Rd. Charlottesville, VA 22903, USA \\ eAstrophysics Group, Cavendish Laboratory, University of Cambridge, JJ Thomson Avenue, \\ Cambridge CB 0HE, UK
}

\begin{abstract}
The Atacama Large Millimeter/submillimeter Array (ALMA) is the world's largest millimeter/submillimeter telescope and provides unprecedented sensitivities and spatial resolutions. To achieve the highest imaging capabilities, interferometric phase calibration for the long baselines is one of the most important subjects: The longer the baselines, the worse the phase stability becomes because of turbulent motions of the Earth's atmosphere, especially, the water vapor in the troposphere. To overcome this subject, ALMA adopts a phase correction scheme using a Water Vapor Radiometer (WVR) to estimate the amount of water vapor content along the antenna line of sight. An additional technique is phase referencing, in which a science target and a nearby calibrator are observed by turn by quickly changing the antenna pointing. We conducted feasibility studies of the hybrid technique with the WVR phase correction and the antenna Fast Switching (FS) phase referencing (WVR+FS phase correction) for the ALMA $16 \mathrm{~km}$ longest baselines in cases that (1) the same observing frequency both for a target and calibrator is used, and (2) higher and lower frequencies for a target and calibrator, respectively, with a typical switching cycle time of $20 \mathrm{~s}$. It was found that the phase correction performance of the hybrid technique is promising where a nearby calibrator is located within roughly $3^{\circ}$ from a science target, and that the phase correction with $20 \mathrm{~s}$ switching cycle time significantly improves the performance with the above separation angle criterion comparing to the $120 \mathrm{~s}$ switching cycle time. The currently trial phase calibration method shows the same performance independent of the observing frequencies. This result is especially important for the higher frequency observations because it becomes difficult to find a bright calibrator close to an arbitrary sky position. In the series of our experiments, it is also found that phase errors affecting the image quality come from not only the water vapor content in the lower troposphere but also a large structure of the atmosphere with a typical cell scale of a few tens of kilometers.
\end{abstract}

Keywords: ALMA, water vapor, atmospheric phase error, phase referencing

\section{INTRODUCTION}

Millimeter/submillimeter interferometers are very powerful tools to investigate emissions from molecular gas and/or dust with very high angular resolutions. The highest spatial resolution is achieved with the longest antenna spacings (baselines). To open up our eyes at millimeter/submillimeter waves, Atacama Large Millimeter/submillimeter Array (ALMA) was constructed at the 5,000 $\mathrm{m}$ in the Atacama desert in Chile where opaque conditions due to the Earth's atmosphere are avoided. Because the most extended array configuration of ALMA has up to $16 \mathrm{~km}$ baselines, the highest spatial resolutions are 60,25, and 17 milli arcseconds at the receiving frequencies of 100,250 , and $350 \mathrm{GHz}$, respectively. With these resolutions, ALMA is expected to reveal unseen astrophysical phenomena in a variety of celestial objects of star forming regions,${ }^{1}$ distant galaxies,${ }^{2}$ small bodies

Ground-based and Airborne Telescopes VI, edited by Helen J. Hall, Roberto Gilmozzi, Heather K. Marshall, Proc. of SPIE Vol. 9906, 99065U · @ 2016 SPIE · CCC code: 0277-786X/16/\$18 · doi: 10.1117/12.2232301 
in the solar system, ${ }^{3}$ and any other astronomical phenomena for which a high spatial resolution is the important key to solve mysteries.

To achieve such high spatial resolutions, an interferometer phase stability taken with the longest baselines is very crucial. ALMA has tested phase correction schemes with baselines as long as possible since its early science operation started in 2011 as the commissioning and science verification phase. ${ }^{4-7}$ In ALMA, a hybrid phase correction scheme using $183 \mathrm{GHz}$ Water Vapor Radiometer (WVR) phase correction ${ }^{8}$ and antenna Fast Switching phase referencing (FS), or WVR+FS phase correction, is thought to be promising for its very long baseline observations. ${ }^{9,10}$ The ALMA WVR+FS phase correction scheme has been successfully demonstrated with the baselines up to $2.7 \mathrm{~km},{ }^{4}$ and it is important to confirm whether this scheme is useful for the maximum baseline. In September to November of each year of 2014 and 2015, ALMA Long Baseline Campaigns (LBCs) were organized to conduct feasibility studies of the imaging capability using its longest baselines of up to $16 \mathrm{~km} .{ }^{11}$ In the LBCs, research of the interferometry phase stability for long baselines was considered to be one of the very important subjects for future advanced science observations with the achievable highest spatial resolutions.

In order to investigate optimum observation parameters for the WVR+FS phase correction, we conducted "two source observation experiments", in other words, FS test experiments in the LBCs. In this report, we introduce the feasibility study results of the ALMA WVR+FS phase correction scheme in the two LBCs (LBC2014 and LBC2015). In Section 2, basic concepts of the hybrid phase correction scheme as well as mathematical expressions of characteristics of the ALMA interferometer phase fluctuations are described. In Section 3, we mention outlines of the FS test experiments. Sections 4 to 6 provide results of our experiments. In Section 7, we provide summaries of this feasibility study.

\section{BASIC CONCEPTS OF ALMA PHASE CORRECTION}

\subsection{WVR+FS phase correction}

The FS phase referencing is expected to effectively reduce phase errors especially for longer baselines. ${ }^{12}$ In the following discussion, a single on-source duration is referred to as a scan. In the FS, alternate scans are made on the target and phase calibrator. One observation period from the beginning of the calibrator scan, then the target scan and return to the beginning of the calibrator scan, is referred to as the switching cycle time, $t_{\mathrm{swt}}$. An arrival time difference of a celestial radio wave between telescopes is largely removed by subtracting the a priori values, calculated in the correlator. We refer to the interferometer phases of the target and phase calibrator as $\phi^{\mathrm{t}}$ and $\phi^{\mathrm{c}}$, respectively, expressed as follows:

$$
\begin{aligned}
\phi^{\mathrm{t}}\left(t^{\mathrm{t}}\right) & =\Phi^{\mathrm{t}}\left(t^{\mathrm{t}}\right)+\epsilon^{\mathrm{t}}\left(t^{\mathrm{t}}\right)-\Phi_{\mathrm{apri}}^{\mathrm{t}}\left(t^{\mathrm{t}}\right)-\Phi_{\mathrm{WVR}}^{\mathrm{t}}\left(t^{\mathrm{t}}\right) \\
& =\Phi_{\mathrm{v}}^{\mathrm{t}}\left(t^{\mathrm{t}}\right)+\Phi_{\text {err }}^{\mathrm{t}}\left(t^{\mathrm{t}}\right)+\epsilon^{\mathrm{t}}\left(t^{\mathrm{t}}\right) \\
\phi^{\mathrm{c}}\left(t^{\mathrm{c}}\right) & =\Phi^{\mathrm{c}}\left(t^{\mathrm{c}}\right)+\epsilon^{\mathrm{c}}\left(t^{\mathrm{c}}\right)-\Phi_{\mathrm{apri}}^{\mathrm{c}}\left(t^{\mathrm{c}}\right)-\Phi_{\mathrm{WVR}}^{\mathrm{c}}\left(t^{\mathrm{c}}\right) \\
& =\Phi_{\mathrm{v}}^{\mathrm{c}}\left(t^{\mathrm{c}}\right)+\Phi_{\text {err }}^{\mathrm{c}}\left(t^{\mathrm{c}}\right)+\epsilon^{\mathrm{c}}\left(t^{\mathrm{c}}\right),
\end{aligned}
$$

where $\Phi_{\text {apri }}$ is the a priori value for $\Phi, \Phi_{\mathrm{WVR}}$ is a phase correction term obtained with the $183 \mathrm{GHz}$ WVR which is installed to each $12-\mathrm{m}$ antenna, $\Phi_{\mathrm{v}}$ is visibility phase component representing a source structure, $\Phi_{\text {err }}$ is a phase error, $\epsilon$ is a thermal noise, $t^{\mathrm{t}}$ is the time when the target is observed, and $t^{\mathrm{c}}$ is the time when the calibrator is observed, temporarily $t_{\mathrm{swt}} / 2$ apart from $t^{\mathrm{t}}$. If the separation angle between the target and calibrator is small enough and the switching cycle time is short enough, $\Phi_{\mathrm{err}}^{\mathrm{t}} \simeq \Phi_{\mathrm{err}}^{\mathrm{c}}$. Assuming that the calibrator is a point source $\left(\Phi_{\mathrm{v}}^{\mathrm{c}}=0\right)$, the WVR+FS phase correction can be conducted as follows:

$$
\phi^{\prime \mathrm{t}}\left(t^{\mathrm{t}}\right)=\Phi^{\mathrm{t}}\left(t^{\mathrm{t}}\right)-\Phi_{\mathrm{apri}}^{\mathrm{t}}\left(t^{\mathrm{t}}\right)-\Phi_{\mathrm{WVR}}^{\mathrm{t}}\left(t^{\mathrm{t}}\right)-\frac{\phi^{\mathrm{c}}\left(t^{\mathrm{t}}-t_{\mathrm{swt}} / 2\right)+\phi^{\mathrm{c}}\left(t^{\mathrm{t}}+t_{\mathrm{swt}} / 2\right)}{2}
$$

where $\phi^{\prime t}\left(t^{t}\right)$ is a WVR+FS-corrected phase of the target. Especially, the separation angle and switching cycle time are considered to be the most important observation parameters to control the performance of the WVR+FS phase correction. 


\subsection{Rms phase, temporal structure function (TSF), and spatial structure function (SSF)}

\subsubsection{Single source observations}

A temporal structure function (TSF) of a two-element interferometer with a baseline vector $\boldsymbol{B}$ is calculated as follows:

$$
\bar{D}_{\phi}(\boldsymbol{B}, p)=<\{\phi(\boldsymbol{B}, t+p)-\phi(\boldsymbol{B}, t)\}^{2}>
$$

where $p$ is a time interval. Let us assume a phase screen and frozen flow for the tropospheric turbulence causing the atmospheric phase fluctuations. ${ }^{13}$ Root mean square (rms) phase of a two-element interferometer $\left(\sigma_{\phi}\right)$ can be expressed using the TSF of a two-element interferometer $\bar{D}_{\phi}(\boldsymbol{B}, t)$ as follows: ${ }^{14}$

$$
\begin{aligned}
\sigma_{\phi}^{2} & =\frac{1}{\tau^{2}} \int_{0}^{\tau}(\tau-t) \bar{D}_{\phi}(\boldsymbol{B}, t) d t \\
& =\frac{1}{\tau^{2}} \int_{0}^{\tau}(\tau-t)\left\{2 D_{\phi}(v t)+2 D_{\phi}(B)-D_{\phi}(|\boldsymbol{v} t+\boldsymbol{B}|)-D_{\phi}(|\boldsymbol{v} t-\boldsymbol{B}|)\right\} d t
\end{aligned}
$$

where $\tau$ is an average time, $\boldsymbol{v}$ is a velocity vector of the phase screen (or a wind velocity aloft), and $D_{\phi}$ is a spatial structure function (SSF) expressed as follows:

$$
D_{\phi}(B)=<2 \pi \nu\{\zeta(\boldsymbol{x}+\boldsymbol{B})-\zeta(\boldsymbol{x})\}^{2}>
$$

where $\nu$ is observing frequency, $\zeta(\boldsymbol{x})$ is an extra phase caused by the excess path of the incident radio wave received by an antenna at $\boldsymbol{x}$. If $v \tau \gg B, \sigma_{\phi}$ can be simplified as $\sqrt{D_{\phi}(B)}$. The wind speed is assumed to be $v=5-20 \mathrm{~m} \mathrm{~s}^{-1}$ for telescope sites. Because ALMA has the longest baselines of $16 \mathrm{~km}$, we can use the above approximation for longer than $\tau \sim 1000 \mathrm{~s}$. In previous studies, the SSF has been treated as a power-law function in many cases as follows:

$$
D_{\phi}(B)=C^{2} B^{\alpha}
$$

where $C$ and $\alpha$ are referred to as a structure coefficient and structure exponent, respectively. For a typical threedimensional (3-D) Kolmogorov turbulence, $\alpha$ is theoretically predicted to be $5 / 3$ for short baselines ( $\leq 1 \mathrm{~km})$ while $\alpha$ is $2 / 3$ for a two dimensional (2-D) Kolmogorov turbulence for long baselines $(\geq 1 \mathrm{~km}) .{ }^{15,16}$

\subsubsection{Phase referencing observations (Two source observations)}

Let us consider the rms phase due to the atmospheric phase fluctuations in the case that two closely located sources are observed. The TSF of the target phase after WVR+FS phase correction can be expressed as follows:

$$
\bar{D}_{\Delta \phi}(\boldsymbol{B}, p)=<\left\{\phi^{\prime \mathrm{t}}(t)\right\}^{2}>
$$

Using the above TSF, the rms phase of the calibrated (phase referenced) target phase $\left(\sigma_{\Delta \phi}\right)$ can be expressed as follows:

$$
\sigma_{\Delta \phi}^{2}=\frac{1}{\tau^{2}} \int_{0}^{\tau}(\tau-t) \bar{D}_{\Delta \phi}(\boldsymbol{B}, t) d t .
$$


In the WVR+FS phase correction, the separation angle between the two sources and $t_{\mathrm{swt}}$ are the most important parameters for successful phase calibration: The smaller the separation angle and/or the shorter the switching cycle time, the better the performance of phase calibration would be. On the hand, it is generally difficult to find a bright and compact calibrator close to an arbitrary target source especially at higher frequencies, and very short switching cycle time increases the over-head time in an observation, which makes the observation efficiency worse. It is required to look for optimum observation parameters for the successful phase calibration in the ALMA science operation.

\section{OBSERVATIONS AND DATA REDUCTION OF FS TEST EXPERIMENTS}

Figure 1 shows one of the array configurations during LBC2014 with the longest baseline length of $\sim 11 \mathrm{~km}$. Twenty to thirty antennas were typically available for our FS test experiments. A pair of bright quasars were observed by turn with the very quick antenna pointing change (antenna fast switching). The on-source duration for each source was about $8 \mathrm{~s}$ while the over-head time for the pointing change was 2 to $4 \mathrm{~s}$ and mainly dependent on the separation angle between the sources. A brighter source of the two was assumed to be a phase calibrator while another fainter one was assumed to be a target. One experiment has about 30 and 20 min in LBC2014 and LBC2015, respectively. Four Base-Band (BB) channel for the two linear polarizations (XX and YY) were correlated every $0.96 \mathrm{~s}$ using the 12-m array correlator with the Time Division Mode.

In the first step of the data reduction of the correlated data, we used CASA (the Common Astronomy Software Applications $)^{17}$ to obtain the WVR-corrected interferometer phase using the offline WVR phase correction software (wvrgcal). ${ }^{18}$ The quantity of the line-of-sight water vapor content in the atmosphere is obtained as Precipitable Water Vapor (PWV), the depth of the water vapor in a column of the atmosphere, using wvrgcal. We also made use of the correlated data without the WVR phase correction, which is referred to as raw interferometer phase in this report.

The frequency-averaged four BB channel data with the bandwidth of $1.875 \mathrm{GHz}$ for the two polarizations were averaged after removing the phase offsets between the BB channels. If we found a problem with the interferometer phase or amplitude with a certain antenna, the baselines with the antennas were flagged out. We obtained antenna-base phase solutions for a target and calibrator independently. We checked the data quality for each antenna solution and flagged out bad data due to troubles in the cross correlation, abnormal WVR outputs, the antenna pointing trouble, and so on. Phase connection was carefully made for the target and calibrator using the antenna-base phase solutions. Each of target and calibrator scans has typically 6 to $8 \mathrm{~s}$ on-source duration, and each scan was averaged in order to reduce the thermal noise contribution. Finally we reconstructed baselinebase phases from the antenna-base solutions which are free from $2 \pi$ ambiguities to calculate SSF using all the available baselines. The same procedure was also applied to the raw interferometer phase except for the wvrgcal application. Table 1 lists examples of totally more than 50 FS test experiments. The left panel of Figure 2 is an example of SSF, that is, rms phase of the baseline-base phase (Band3) as a function of baseline length obtained from No.2 experiment. The black and red open circles represent the rms phases of the raw and WVR-corrected interferometer phases, respectively.

\section{RESULTS OF FS TEST EXPERIMENTS}

For the FS phase referencing, we used a simple algorithm to interpolate a target source phase from calibrator phases of two calibrator scans sandwiching the target source scan. Note that a phase offset for each of the antenna-base phase time series is removed but a linear trend is not. In order to investigate the dependency of the phase correction scheme on the switching cycle time using the same data, the calibrator scans were flagged to simulate longer switching cycle time.

SSF of the WVR+FS-corrected phase is shown in the left panel of Figure 2 with orange and blue crosses for the switching cycle times of 18.9 and 115.9 s, respectively, for a pair of sources J2000-1748 and J2005-1822 with the separation angle of $1.2^{\circ}$. SSF with a $10 \mathrm{~km}$ baseline decreases with a factor of 10 (1 to $0.1 \mathrm{~mm} \mathrm{rms}$ in excess path length) using the WVR+FS phase correction with the switching cycle time of $20 \mathrm{~s}$, so that the hybrid phase correction scheme was proved to be quite promising for long baselines. We revisit the performance 

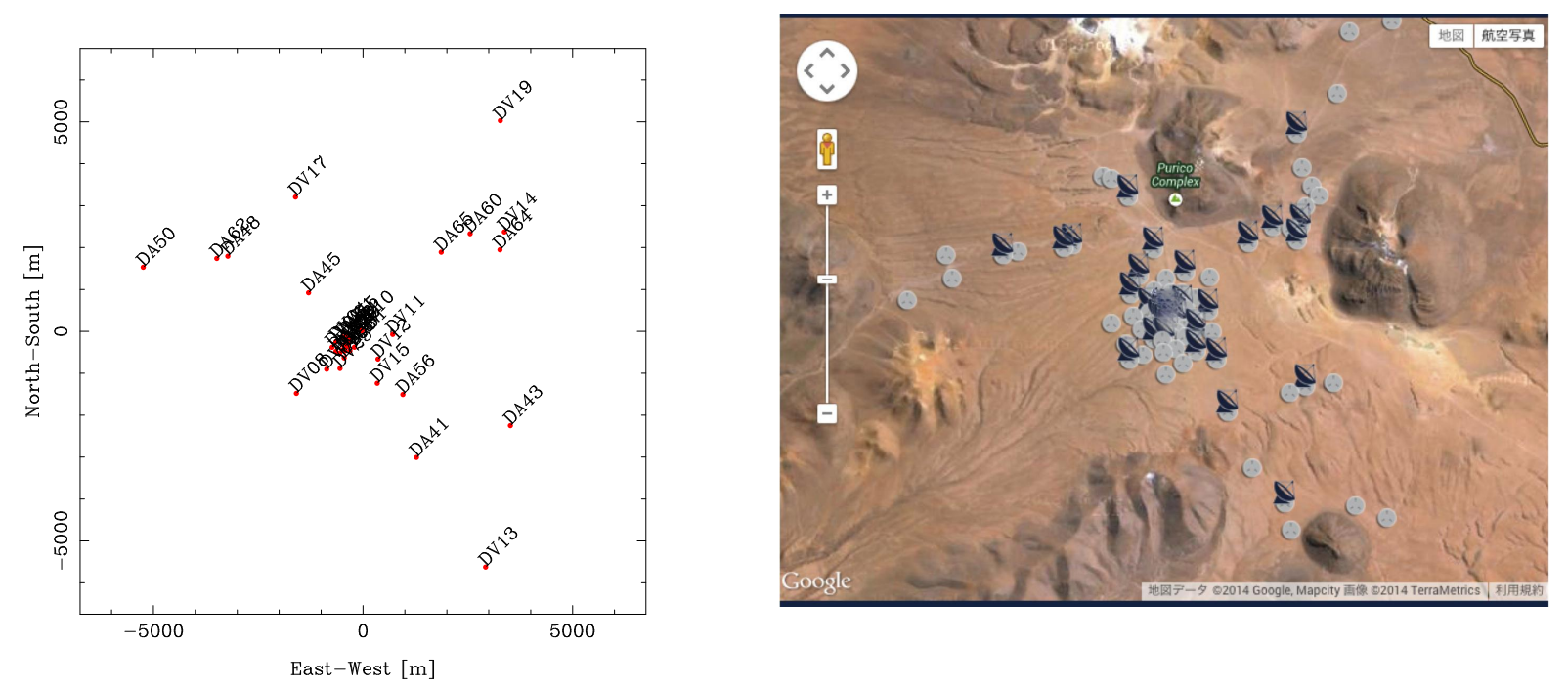

Figure 1: Example of the array configuration during LBC2014 (24th of September, 2014) and its sky-view (http: //www.almaobservatory.org/en/alma-from-the-sky). In the left panel, "DA $N N$ " and "DV $N N$ " represent the ALMA 12-m antenna identification name. The four eastern cluster antennas (DV14, DA60, DA64, and DA65) were located between Cerro Chajnantor and Cerro Chascon. DV13 at the most southern position was located between two altitude peaks as well.

Table 1: Examples of the FS experiments

\begin{tabular}{rlrccccc}
\hline \hline No. & Date & Time (UT) & $\begin{array}{c}\text { Source 1 } \\
(\text { EL }[\mathrm{deg}])\end{array}$ & $\begin{array}{c}\text { Source 2 } \\
(\text { EL }[\mathrm{deg}])\end{array}$ & $\begin{array}{c}\text { Sep. angle } \\
{[\mathrm{deg}]}\end{array}$ & $\begin{array}{c}\text { PWV } \\
{[\mathrm{mm}]}\end{array}$ & Band \\
\hline 1 & Sep 19, 2014 & $07: 59-08: 26$ & J0739+0137 (25.4) & J0745-0044 (25.0) & 2.9 & 0.34 & {$[3,3]^{1}$} \\
2 & Sep 24, 2014 & $02: 27-02: 53$ & J2000-1748 (57.2) & J2005-1822(58.3) & 1.3 & 0.90 & {$[3,3]^{1}$} \\
3 & Oct 3, 2014 & $04: 08-04: 35$ & J2000-1748 (25.7) & J2011-1546(27.4) & 3.2 & 5.49 & {$[3,3]^{1}$} \\
\hline 4 & Oct 11, 2014 & $22: 36-23: 03$ & B2245-328(47.5) & J2302-3718(44.5) & 5.5 & 1.66 & {$[3,3]^{1}$} \\
5 & Oct 11, 2014 & $23: 47-00: 14$ & J2250-2806 (62.5) & B2255-282(60.9) & 1.6 & 1.77 & {$[3,3]^{1}$} \\
6 & Oct 12, 2014 & $00: 16-00: 42$ & J2345-1555(55.0) & B2345-167(54.5) & 0.9 & 1.61 & {$[3,3]^{1}$} \\
7 & Oct 12, 2014 & $00: 43-01: 10$ & B2325+093 (49.2) & J2334+0736(49.8) & 2.6 & 1.21 & {$[3,3]^{1}$} \\
8 & Oct 12, 2014 & $01: 38-02: 06$ & B2246+208 (45.9) & J2253+161 (50.8) & 5.1 & 1.12 & {$[3,3]^{1}$} \\
\hline 9 & Oct 12, 2015 & $02: 27-02: 46$ & J0049-4457(62.2) & J0056-4451(61.3) & 1.3 & 1.23 & {$[7,3]^{2}$} \\
10 & Oct 12, 2015 & $03: 09-03: 29$ & J0207-3857 (60.2) & J0200-4010(60.9) & 3.5 & 1.28 & {$[7,3]^{2}$} \\
\hline \hline
\end{tabular}

${ }^{1}$ The reference frequency in Band3 is $86.2 \mathrm{GHz}$.

${ }^{2}$ The reference frequencies in Band7 and Band3 are 278.9 and $89.0 \mathrm{GHz}$, respectively. 
of the hybrid phase correction scheme for ALMA in Section 6 to investigate criteria of the separation angle and switching cycle time more precisely.
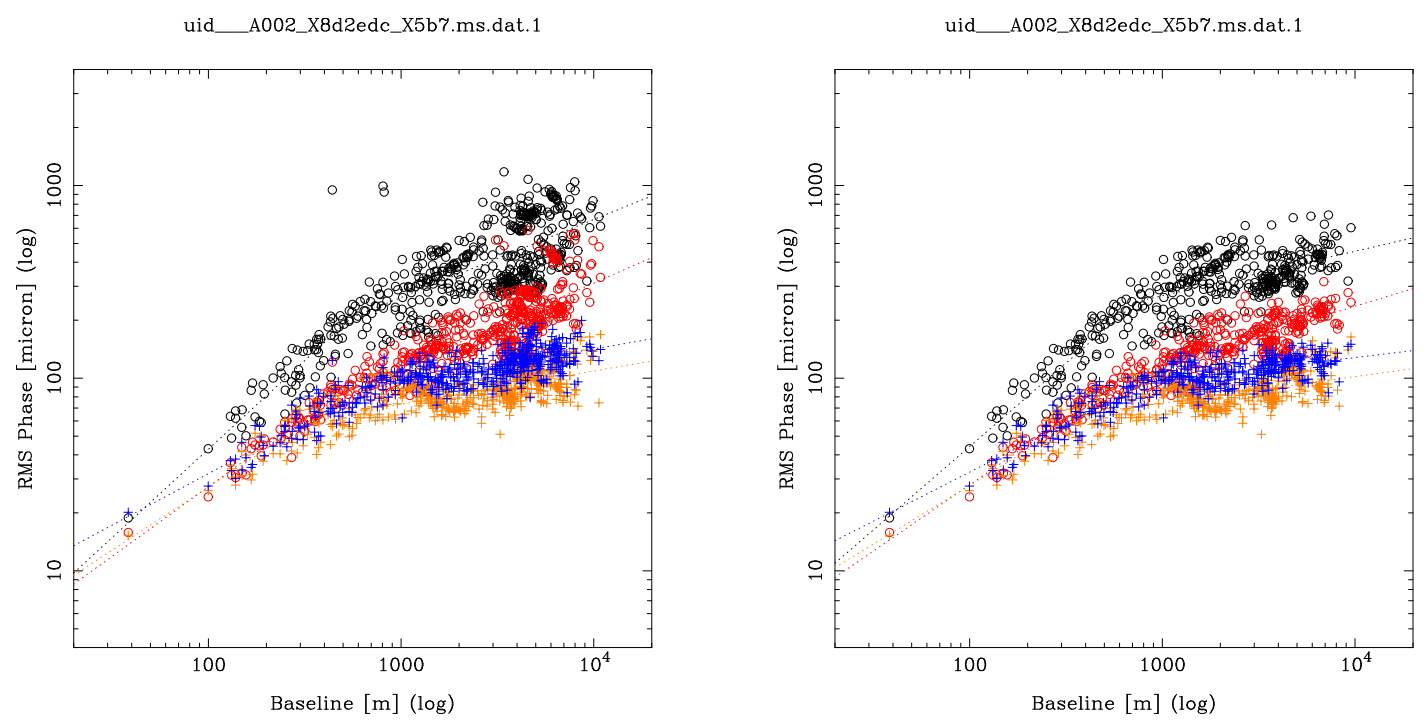

Figure 2: SSF of No.2 experiment for a pair of bright sources (J2000-1748 and J2005-1822) with the separation angle of $1.2^{\circ}$. Black and red open circles represent the rms phase of the raw and WVR-corrected interferometer phase of J2005-1822, and orange and blue crosses represent the rms phase of the WVR+FS-corrected data with the switching cycle time of 19 and $116 \mathrm{~s}$, respectively. The horizontal axis is baseline length, and the vertical axis is rms phase expressed in excess path length in micron. Left: All the antennas are used in calculating SSF. Right: The eastern cluster antennas (DV14, DA60, and DA64) and DV13 are excluded in calculating SSF.

\subsection{Understanding of SSF at the ALMA site}

It is noted that there are prominent outliers in SSF of the raw interferometer phase with the baseline length of 0.4 and $1 \mathrm{~km}$ in the left panel of Figure 2. Here we refer to four antennas in the way of the eastern arm (DV14, DA60, DA64, and DA65 in Figure 1) as "eastern cluster antennas". Figure 3 shows the time series of the raw and WVR-corrected interferometer phases of the baselines between the three eastern cluster antennas (DA60, DA64, and DV14) of No.2 experiment. The baseline length ranges $0.4-1 \mathrm{~km}$ among the three. On the other hand, Figure 3 shows the phase time series of much longer baselines between one of the central cluster (DV10) and the furthest antennas of DA50, DV19, and DV13 whose baseline lengths are $\sim 5 \mathrm{~km}$. Comparing these two figures, phase fluctuations among the eastern cluster antennas are much larger than those with the $5 \mathrm{~km}$ baselines. Such large phase fluctuations with rather short baselines is referred to as "anomalous phase fluctuations" in this report. These anomalous phase fluctuations in the raw interferometer phases can be significantly reduced using the WVR phase correction as shown in the right panel of Figure 3. This suggests that the anomalous phase fluctuations come from not instrument troubles (frequency standard etc.) but the water vapor disturbances in the atmosphere. The sky-view in Figure 1 (right) displays that the eastern cluster antennas were located between two high mountains of Cerro Chajnantor (alt.5,604 m) and Cerro Chascon (alt.5,698 m). Such a geometry may induce strong turbulences because the two mountains can disturb wind flows over the ALMA site.

In the same experiment (No.2), DV13 (the most southern antenna as shown in Figure 1) had an abrupt phase change at around 2:49 - 2:52 UTC as shown in the middle line of Figure 4. This remarkable change makes the phase stabilities of baselines with DV13 worse than those of the other long baselines, and can be also significantly reduced using the WVR phase correction as shown in the right panel of Figure 4. DV13 was located close to mountains as shown in the sky-view, so that this antenna might have a similar situation to the eastern cluster antennas. The right panel of Figure 2 shows SSF after excluding the eastern cluster antennas and DV13. It can be easily noticed that the outliers for the raw interferometer phase disappeared in SSF. This revised SSF shows 
us typical characteristics of the atmospheric phase fluctuations which can be understood with the Kolmogorov turbulence and the frozen flow screen model of the lower troposphere. ${ }^{15,19}$

In the LBCs, we have often seen the anomalous phase fluctuations for specific antennas closely located to the mountains, which can be improved using the WVR phase correction. From the atmospheric science view point, it is worth while investigating these phenomena more precisely to clarify the characteristics of the ALMA site. In this report, we did not exclude those antennas showing the anomalous phase fluctuations in our data analysis with SSF. In the following discussions, we adopt the two component power-law model for the SSF with the 3-D and 2-D Kolmogorove turbulences as described in Section 2.2.1 although the power-laws are free parameters to be fitted. The model fitting was conducted for each experiment. Figure 2 shows the model fitting results with the dotted lines. Statistical analysis of SSF for ALMA single source observations is reported more precisely in another SPIE paper. ${ }^{7}$
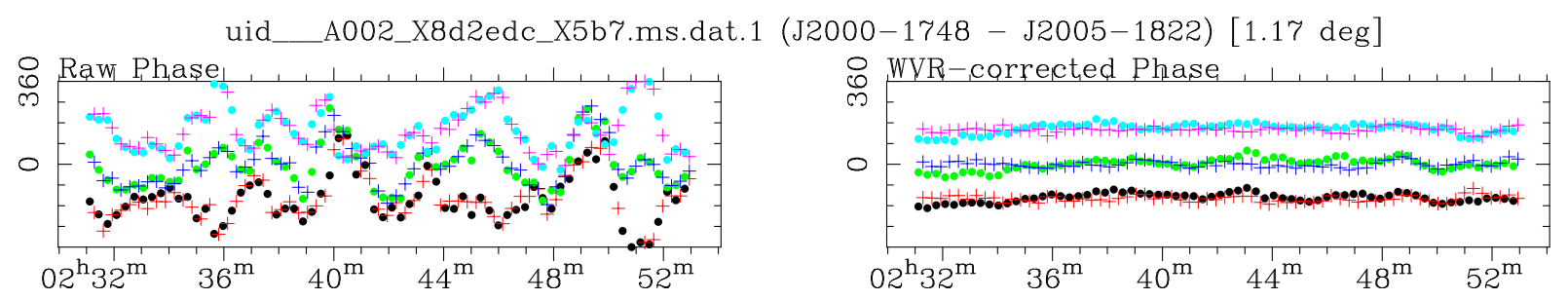

Figure 3: Time series of the interferometer phase of No.2 experiment between the antennas of DA60, DA64, and DV14 (eastern cluster antennas). The left and right panels show the raw and WVR-corrected interferometer phases, respectively. In each time series, the filled circles and crosses represent target and calibrator phases, respectively. Phases of two sources observed with the fast switching are shown in the same panels.
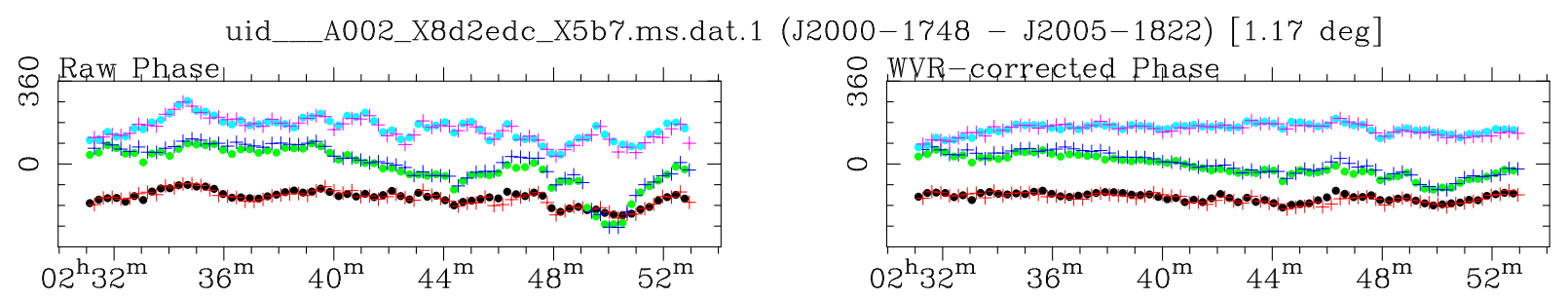

Figure 4: Time series of the interferometer phase of $5 \mathrm{~km}$ baselines of No.2 experiment. The top, middle, and bottom lines show the time series of DV19, DV13, and DA50, respectively, with respect to DV10 at the array center.

\subsection{Statistics of performance of the WVR phase correction}

Figure 5 shows histograms of an improvement factor of the WVR phase correction which is defined by a ratio of the rms phase between before and after the WVR phase correction. The improvement factor larger than unity means successful WVR phase correction. The histograms indicate that the WVR phase correction is successful for almost all the experiments more or less with some exceptions: The orange bars in the figure represent experiments with the unsuccessful WVR phase correction. We infer that roughly $5 \%$ of science observations may not be improved by the WVR phase correction where the weather conditions are judged to be suitable to run observations. Figure 6 shows the time series of the raw and WVR-corrected interferometer phases of No.3 experiment as listed in Table 1. This is a typical case that the WVR phase correction makes the phase stability worse. We can empirically categorize the ineffective and unsuccessful WVR correction cases into the following two:

- Ineffective WVR phase correction under low PWV situation (PWV $\leq 0.5 \mathrm{~mm})$ :

Because the water vapor is very little in the atmosphere, it does not contribute to the excess path length, 
and the WVR phase correction can hardly improve the phase stability. Note that the improvement factor is greater than unity, so that there is no reason to stop the WVR phase correction in this case.

- Unsuccessful WVR phase correction under high PWV situation (PWV $\geq 3 \mathrm{~mm}$ ):

Because there is non-vapor water contents (for example, clouds), the WVR can sense not only the water vapor but also radiation from the liquid water and/or scattered radiation by the ice. In this case, the WVR phase correction makes the phase stability worse. In No.3 experiment, PWV was estimated to be $5.4 \mathrm{~mm}$ in wrrgcal.

The improvement factor can be easily evaluated from a few minute bright source observations by comparing the rms phases before and after the WVR phase correction and has been monitored in the ALMA science observation operations.
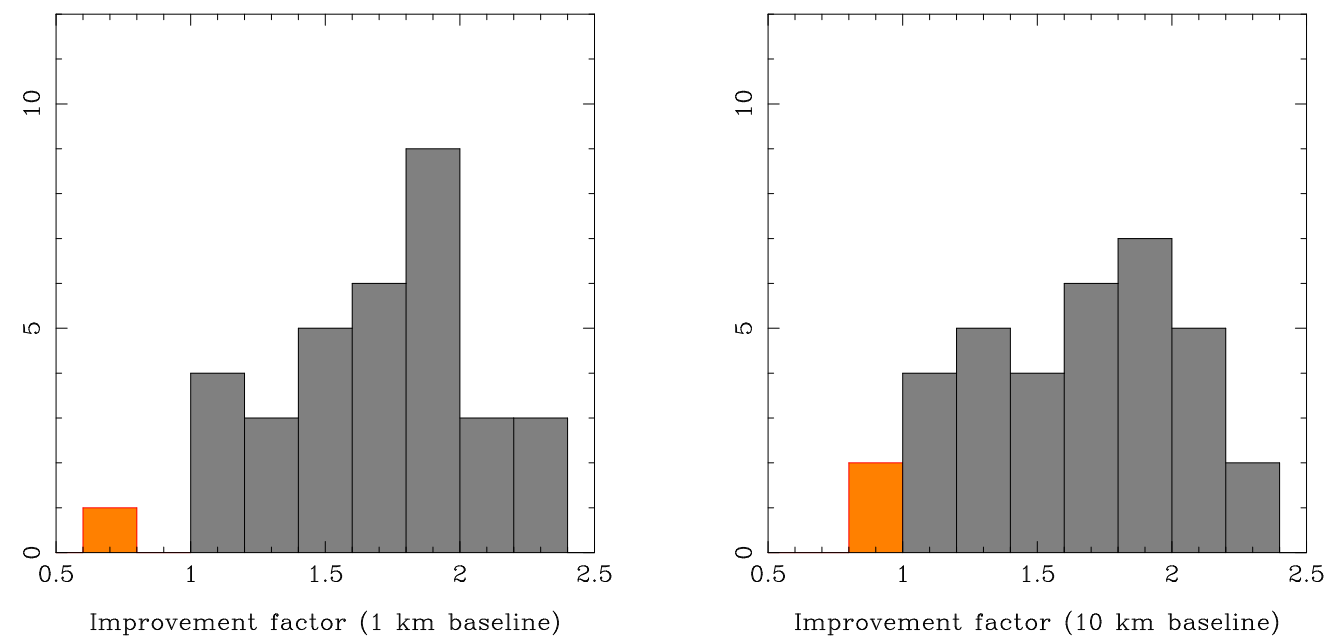

Figure 5: Histograms of the improvement factor of the WVR phase correction. Left: $1 \mathrm{~km}$ baseline case. Right: $10 \mathrm{~km}$ baseline case. Orange bars are experiments with the improvement factor less than unity.
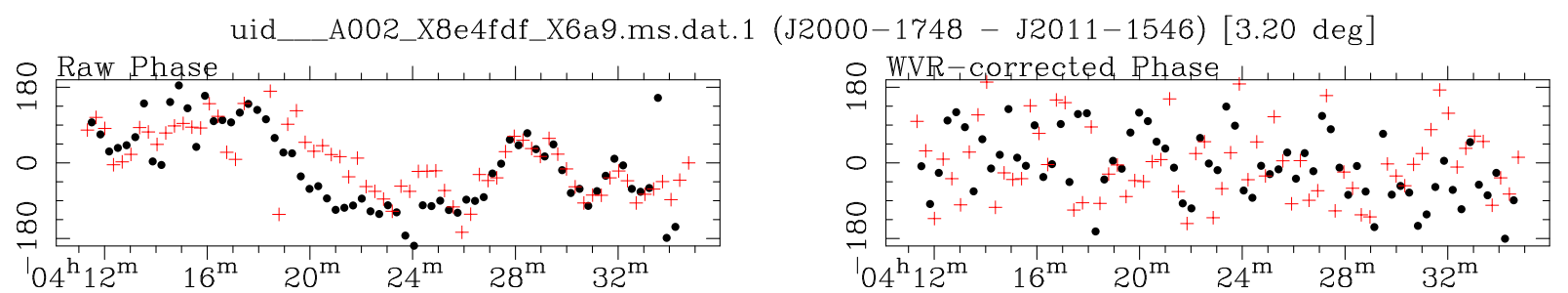

Figure 6: Time series of the interferometer phase of a $2.2 \mathrm{~km}$ baseline (DV54-DV12) of No.3 experiment. The left and right panels show the time series before and after the WVR phase correction, respectively. The black filled circle and red cross represent the averaged target and calibrator scans, respectively.

\subsection{Exceptional cases that the FS phase referencing does not work}

Some of the experiments suggest that the FS phase referencing does not work due to unknown reasons. Figures 7 and 8 shows the time series of the raw and WVR-corrected interferometer phases of No.5 experiment as an example of the case. In the experiment, we selected a pair of sources (J0739+0137 and J0745-0044) with the separation angle of $2.9^{\circ}$. Because the PWV was quite low during the experiment $(0.34 \mathrm{~mm})$, the WVR phase correction is not effective as already mentioned in Section 4.2. Figure 8 shows the phase referencing result of the raw and WVR-corrected interferometer phases. An abrupt change of the target (J0745-0044) interferometer 
phase happened 2-3 min prior to the similar time variation in the calibrator (J0739+0137) interferometer phase. Although FS could reduce short-term phase fluctuations, long-term time variations were inversely added to the target phase by differentiating the phases. In this case, neither of the WVR phase correction nor the FS phase referencing is very useful for the phase correction.

We investigated phase delays of the four BBs at the reference frequencies of 86.2,88.1,96.4, and $98.3 \mathrm{GHz}$ in Band3 and found that the delays are proportional to the reference frequency. This suggests that the long-term phase variations are caused not in the ionosphere but in the troposphere. ${ }^{20}$ The WVR phase correction could not reduce the phase fluctuations, so that the time variations mostly did not come from the water vapor. Our question is why the phase referencing does not work for the separation angle of $2.9^{\circ}$. One possibility is that there were blobs with different reflective indexes which horizontally distributed further than kilometers away in the direction of one source. Because the elevation angle was at around $25^{\circ}$ in the experiment, the horizontal difference between the sources could be a few kilometers, it might happen that the blobs traveled across the pass between the another source and a 12-m antenna a few minutes after they passed the first one.
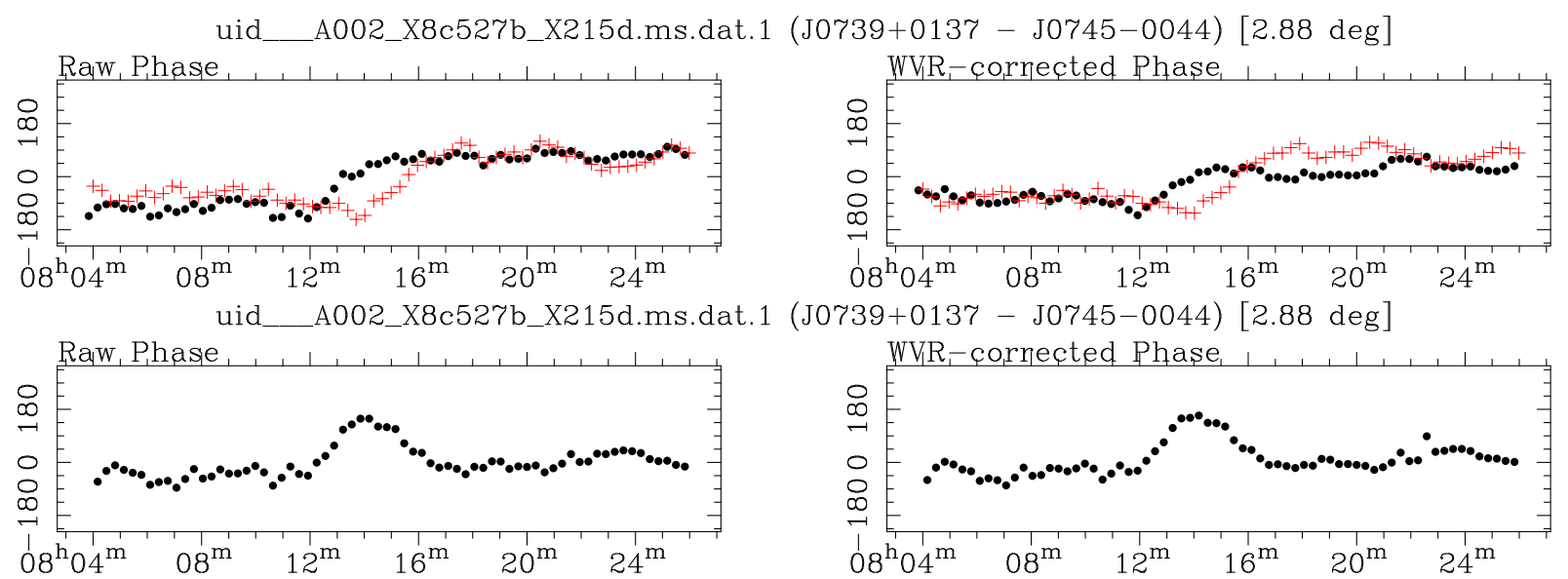

Figure 7: Time series of the interferometer phase of the target and calibrator of No.3 experiment DA43-DV08 baseline. The black filled circle and red cross represent averaged target and calibrator scans, respectively. The left and right panels show the phases with and without the WVR phase correction, respectively. The top and bottom panels show the time series before and after the FS phase referencing.

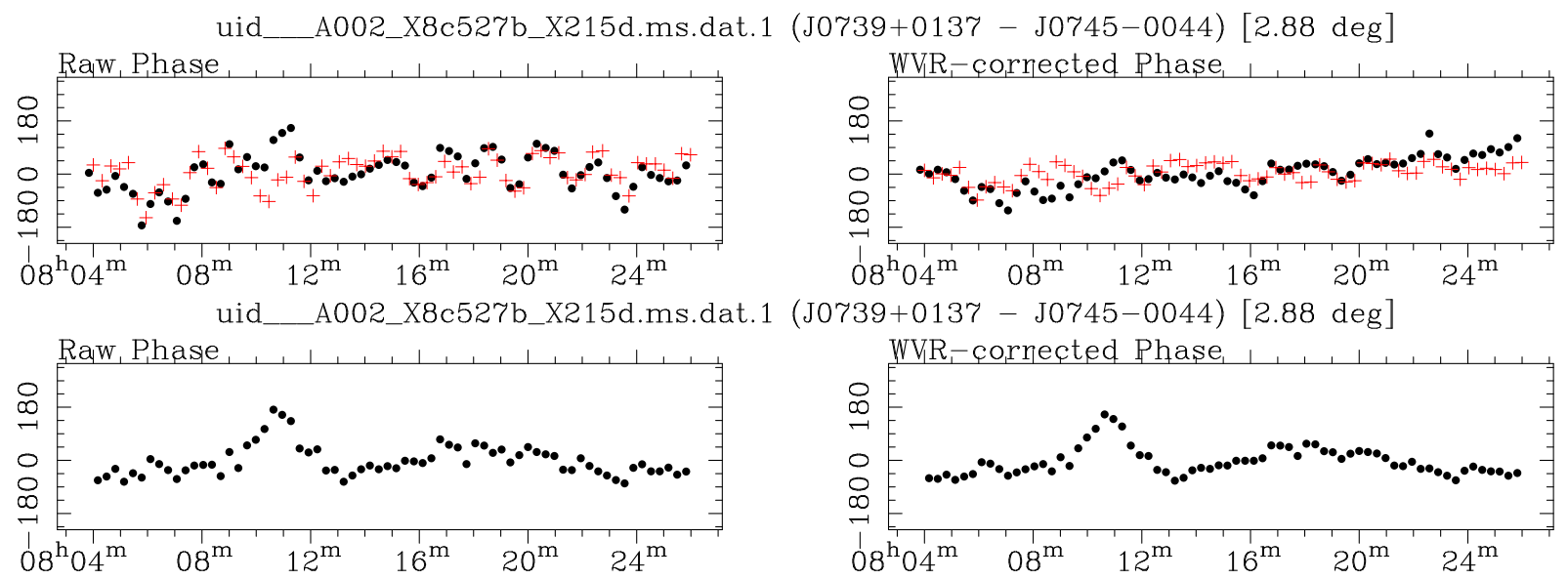

Figure 8: The same as Figure 7 but the baseline of DA60-DV14 (between the eastern cluster antennas). 


\section{BAND-TO-BAND PHASE TRANSFER PHASE REFERENCING $(\mathrm{WVR}+\mathrm{FS}+\mathrm{B2B})$}

Although the WVR+FS phase correction is promising for an advanced ALMA phase correction especially for the long baselines, FS has difficulty to be applied at higher frequencies because it is not easy to to find a bright and compact calibrator around an arbitrary sky position within a few degrees. A possible solution is to observe a phase calibrator at lower frequency where a large number of bright phase calibrators are available. The refractive index of the water vapor is almost nondispersive in the observed frequency range covered with ALMA ${ }^{20}$ so that the calibrator phase at lower frequency can be applied by multiplying a frequency ratio between the target and calibrator. ${ }^{9}$ This technique is called "Band-to-Band phase transfer", or just "B2B". The first B2B experiments were arranged with Noveyama Millimeter Array between 19.5 and $146 \mathrm{GHz}$ and successfully demonstrated to improve the phase stability of the high frequency interferometer phase. ${ }^{21}$ In imaging with Very Long Baseline Interferometry, the B2B technique is successfully applied to continuum sources from 22 to $86 \mathrm{GHz},{ }^{22}$ and $\mathrm{H}_{2} \mathrm{O}$ maser line at $22 \mathrm{GHz}$ to $\mathrm{SiO}$ maser line at $43 \mathrm{GHz} .{ }^{23}$

In LBC2015 we conducted FS test experiments for the feasibility study of he WVR+FS+B2B phase correction with ALMA. The data reduction process is the same as the WVR+FS phase correction, but the calibrator phase is scaled up to the target observing frequency with the frequency ratio. Figure 9 shows the time series of the raw and WVR-corrected interferometer phases of No.9 experiment listed in Table 1. The calibrator phase (red cross) is well aligned with the target phase (black filled circle). SSF of the WVR+FS+B2B-corrected interferometer phase with $t_{\mathrm{swt}}$ of 20 and $120 \mathrm{~s}$ as well as of the raw and WVR-corrected interferometer phases are shown in Figure 10 for No.9 and No.10 experiments. The performance with the B2B technique does not show any prominent difference from the WVR+FS phase correction, so that the WVR+FS+B2B phase correction scheme is expected to be promising for high frequency ALMA observations with long baselines.
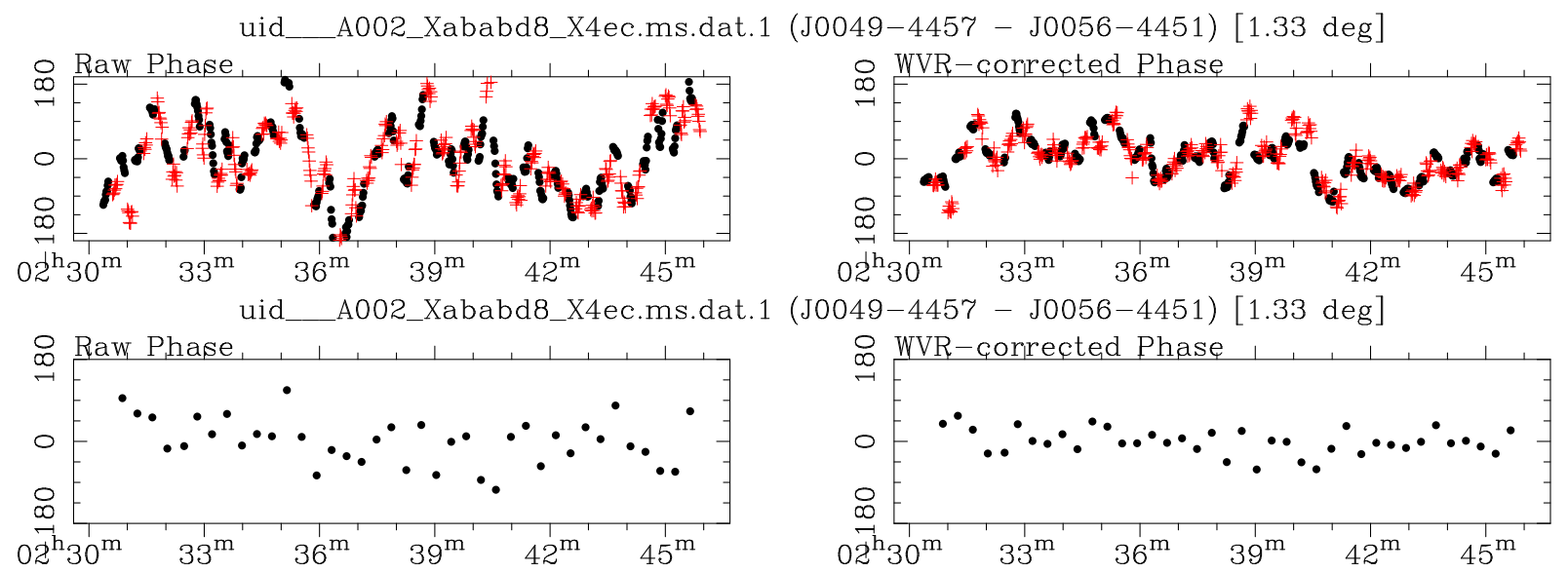

Figure 9: Top: Time series of target Band7 (black filled circle) and calibrator Band3 (red cross) interferometer phases of $10 \mathrm{~km}$ baseline of No.9 experiment. The Band3 interferometer phase is multiplied by the frequency ratio between the Band7 and Band3 reference frequencies. Note that the scans are not averaged, so that each point show $0.96 \mathrm{~s}$ correlator accumulated period data. Bottom: Target phase in Band7 after the FS phase referencing. Note that the target scan is avereged.

\section{CRITERIA OF SEPARATION ANGLE AND SWITCHING CYCLE TIME FOR SUCCESSFUL WVR+FS $(+\mathrm{B} 2 \mathrm{~B})$}

\subsection{Rough estimation of criteria of separation angle and switching cycle time in $\mathbf{W V R}+\mathbf{F S}(+\mathbf{B} 2 \mathrm{~B})$}

There are not a small number of experiments that the WVR+FS as well as WVR+FS+B2B with the switching cycle time of $20 \mathrm{~s}$ can improve the phase stability of the interferometer phase where a closely located calibrator 

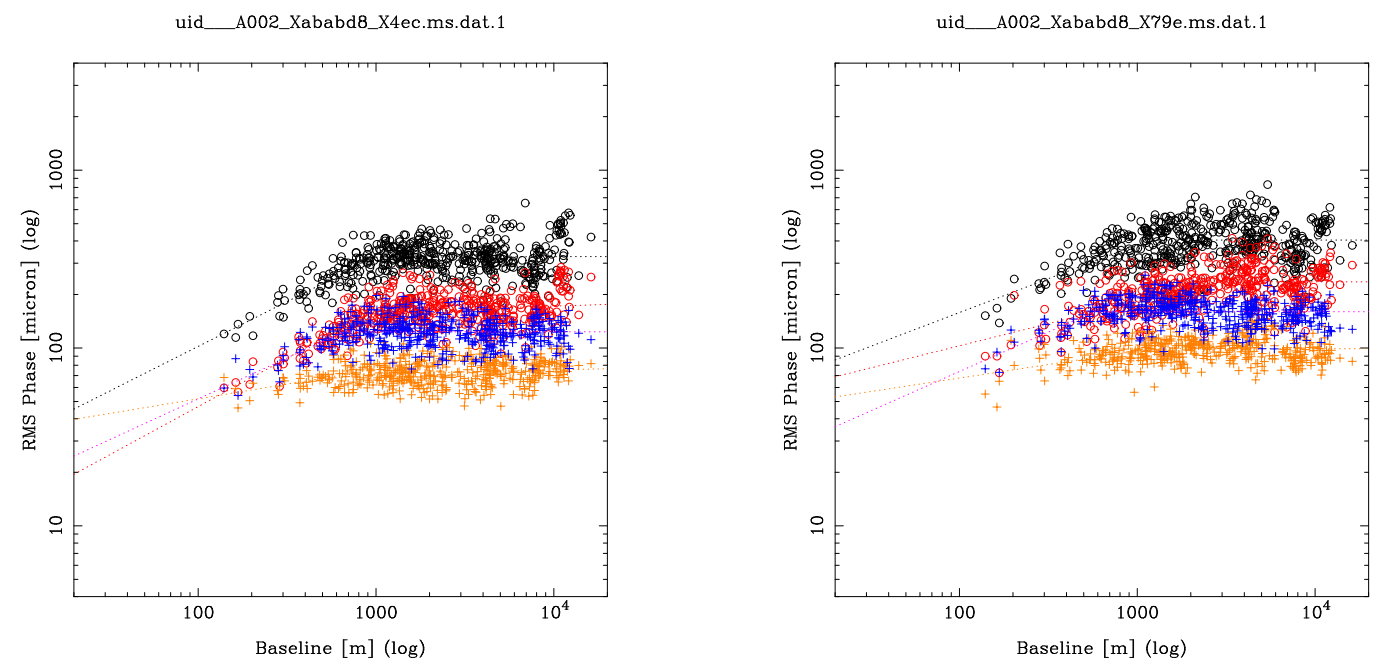

Figure 10: The same as Figure 2 but of of the WVR+FS+B2B-corrected interferometer phase (Band3 $\rightarrow$ Band7) of No.9 (left) and No.10 (right) experiments listed in Table 1.

from a science target is used. So far, there have been 48 available samples (successful experiments without system troubles and/or severe weather conditions) in the two LBCs including the experiments listed in Table 1 but No.3 experiment due to the high PWV condition. We here try to obtain some guidelines in terms of the separation angle and switching cycle time for the advanced ALMA phase correction. For the following discussion, we here adopt "rms phase ratio" $R_{\phi_{\mathrm{rms}}}$ defined as follows:

$$
R_{\phi_{\mathrm{rms}}}=\frac{\mathrm{WVR}+\mathrm{FS}(+\mathrm{B} 2 \mathrm{~B}) \text {-corrected rms phase }}{\text { WVR-corrected rms phase }}
$$

so that $R_{\phi_{\mathrm{rms}}}$ less than unity indicates that the FS phase referencing effectively works. Figure 11 shows the rms phase ratio as a function of separation angle for the switching cycle time of $20 \mathrm{~s}$ and $120 \mathrm{~s}$ in the left and right panels, respectively, and Figure 12 shows the enlarged bottom left corner of Figure 11 in order to focus on smaller separation angles. Although the deviation is not small because all the available samples obtained under various atmospheric conditions ( $\mathrm{PWV}$, sky position, weather condition, etc.) were compiled, we can find a trend in the panels that, smaller the separation angle is, the more effective the WVR+FS $(+\mathrm{B} 2 \mathrm{~B})$ phase correction becomes. Although it is difficult to make a concrete criterion in terms of the separation angle from the figures, we can averagely obtain $R_{\phi_{\mathrm{rms}}} \leq 0.5$ where the separation angle is $\sim 3^{\circ}$ or smaller. Especially, with the above separation angle criterion, the switching cycle time of $20 \mathrm{~s}$ yields greater advantage in the performance comparing with the switching cycle time of $120 \mathrm{~s}$. On the other hand, where a calibrator is separated further than $3-4^{\circ}$, a rather longer switching cycle time barely degrades the performance. The phase stability of the $10 \mathrm{~km}$ baseline can be improved more effectively than that of the $1 \mathrm{~km}$ baseline in terms of the rms phase ratio. However, even with a rather short baselines $(1 \mathrm{~km})$, the phase stability can be greatly improved with the shorter switching cycle time.

\subsection{Where the atmospheric phase fluctuations are caused?}

As mentioned above, we found a statistical trend to constrain the optimal observation parameters such as the separation angle and switching cycle time for the successful WVR+FS $(+\mathrm{B} 2 \mathrm{~B})$ phase correction. On the other hand, because the atmospheric conditions are different day by day, we are not successful to construct a physical model to correctly predict the performance. In order to understand the mechanism of the hybrid phase correction scheme more analytically, we arranged special experiment series in LBC2014 (No.4 to No.8 listed in Table 1) in which source pairs with different separation angles were observed at almost the same elevation angle $\left(50-63^{\circ}\right)$ within a few hours under the same weather condition. The separation angles of the five pairs are $0.9^{\circ}, 1.6^{\circ}$, 
20-s Switching

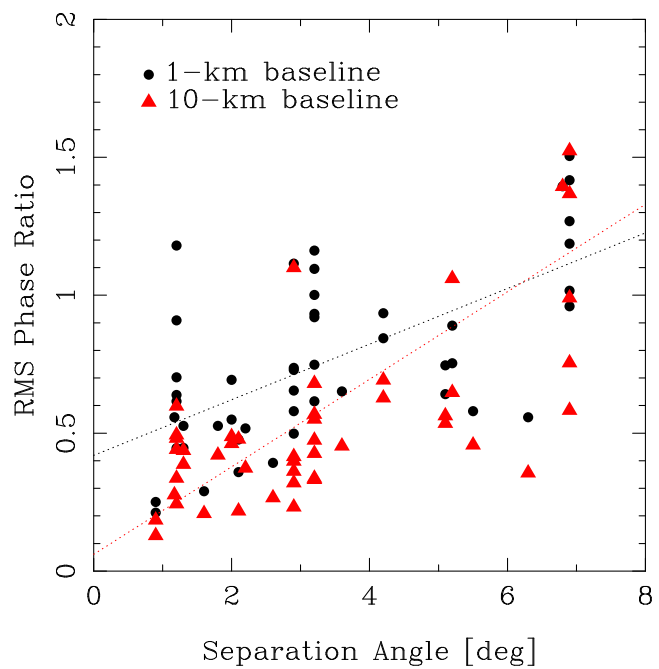

$120-$ s Switching

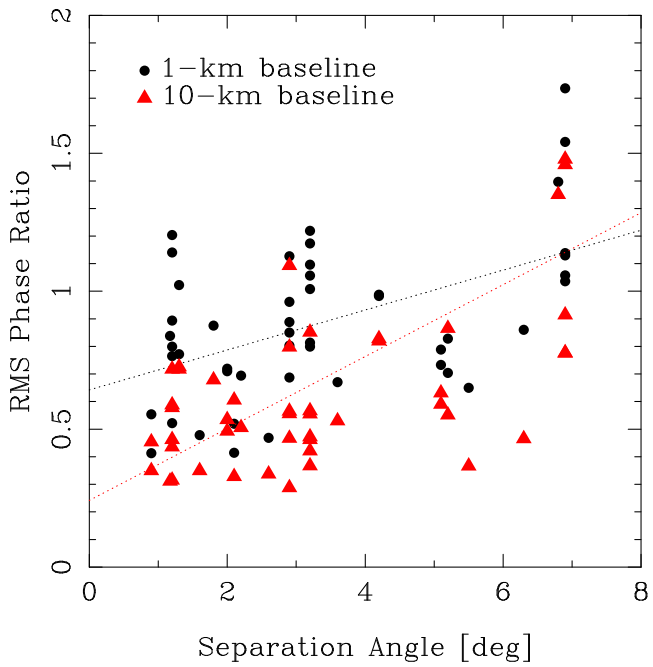

Figure 11: Rms phase ratio as a function. The horizontal axis is the separation angle. The filled black circles and red triangles represent the rms phase ratio for the baseline of 1 and $10 \mathrm{~km}$, respectively. The dotted lines are the linear trend fitting Left: Switching cycle time of $20 \mathrm{~s}$. Right: Switching cycle time of $120 \mathrm{~s}$.

20-s Switching

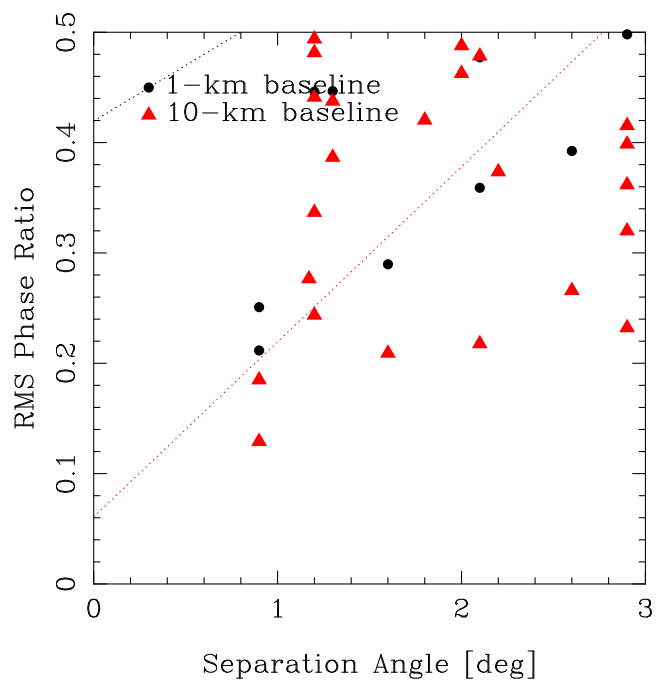

120-s Switching

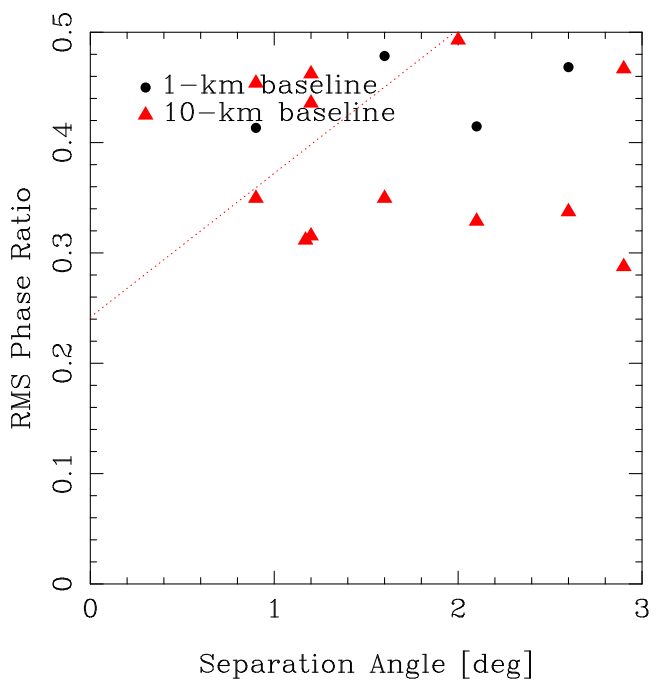

Figure 12: The same as Figure 11, but enlarged for the separation angle smaller than $3^{\circ}$ 
$2.6^{\circ}, 5.1^{\circ}$, and $5.5^{\circ}$. Figure 13 shows SSF of the raw, WVR-corrected, and WVR+FS-corrected interferometer phases.

Before looking at the result of the separation angle dependency of the performance of the day, let us consider the analytical relationship of the rms phase of the WVR+FS-corrected phase. From previous studies, the rms phase of the WVR+FS-corrected phase for long baselines is almost independent of the baseline length and can be expressed as follows: ${ }^{24}$

$$
\begin{aligned}
\sigma_{\Delta \phi}[\mathrm{m}] & \approx \sqrt{2} C_{\mathrm{n}} \sqrt{1.4 H_{\mathrm{s}}} \sqrt{\sec Z_{\mathrm{g}}} \\
& \times\left(\frac{v_{\mathrm{w}} t_{\mathrm{swt}}}{2}+H_{\mathrm{w}} \Delta \theta \sec Z_{\mathrm{g}}\right)^{\frac{\alpha}{2}},
\end{aligned}
$$

where $C_{\mathrm{n}}$ is the structure coefficient of the SSF of the troposphere to the zenith ${ }^{12}$ after the WVR correction, $H_{\mathrm{s}}$ is the scale height of the tropospheric water vapor, $v_{\mathrm{w}}$ is the wind velocity aloft or flow speed of the phase screen, $H_{\mathrm{w}}$ is the height of the phase screen, $\Delta \theta$ is the separation angle between two sources in radian, $\alpha$ is the 3 -D turbulence structure exponent, and $Z_{\mathrm{g}}$ is the zenith angle. In the case of the above arranged experiments, $\alpha$ ranges 1.24-1.56. Assuming $v_{\mathrm{w}}=15 \mathrm{~m} \mathrm{~s}^{-1}$ and $t_{\mathrm{swt}}=20 \mathrm{~s}\left(v_{\mathrm{w}} t_{\mathrm{swt}} / 2=150\right)$ and $\alpha=1.4$ (the median value of No.4 to 8 experiments), we can express Eq.(11) as follows:

$$
\frac{\sigma_{\Delta \phi}}{\sqrt{\sec Z_{\mathrm{g}}}} \approx A\left(150+H_{\mathrm{w}} \Delta \theta \sec Z_{\mathrm{g}}\right)^{0.7}
$$

where $A=\sqrt{2} C_{\mathrm{n}} \sqrt{1.4 H_{\mathrm{s}}}$. In addition, assuming $H_{\mathrm{w}}$ has a typical height of $1.5 \mathrm{~km}$, Eq.(12) is applicable for a baseline longer than $1 \mathrm{~km}$, that is, $\sigma_{\Delta \phi}$ is independent of the baseline length.

Figure 14 shows $\sigma_{\Delta \phi}$ as a function of separation angle for $1 \mathrm{~km}$ and $10 \mathrm{~km}$ baseline cases. The rms phase in Figure 14 can be fitted to the following relation:

$$
\begin{aligned}
\left.\sigma_{\Delta \phi} \text { [micron }\right] & \sim 13+13 \times\left(\frac{180 \times \Delta \theta}{\pi}\right) \quad \text { for } 1 \mathrm{~km} \text { baseline } \\
& \sim-10+36 \times\left(\frac{180 \times \Delta \theta}{\pi}\right) \quad \text { for } 10 \mathrm{~km} \text { baseline }
\end{aligned}
$$

From the above model fitting using Eq.(12) and the assumption of the altitude of the thin phase screen of $1.5 \mathrm{~km}$, we obtain $A=1.80 \times 10^{-2} \mathrm{~m}^{0.3}$ and $A=2.59 \times 10^{-2} \mathrm{~m}^{0.3}$ for the baseline length of 1 and $10 \mathrm{~km}$, respectively. The above fitting result is not easy to be understood because, being different from the above prediction, $\sigma_{\Delta \phi}$ is clearly dependent not only on the separation angle but also the baseline length.

In the top two panels of Figure 13, SSF of the WVR+FS-corrected phase for the separation angles of $0.9^{\circ}$ and $1.6^{\circ}$ slightly increases with the baseline length for longer than several $\mathrm{km}$. For the separation angle of $2.6^{\circ}$ (middle left panel in Figure 13), the power-law slope of SSF of the WVR+FS-corrected phase is steeper than those of the smaller separation angles. For the separation angles of $5.1^{\circ}$ and $5.5^{\circ}$ (middle right and bottom), SSF has clear dependency on the baseline length, so that there seems to be another power-law component in SSF as a function of baseline length after the WVR+FS phase correction.

In order to quantitatively explain Figure 14, we tried to adopt an additional thin phase screen with the 3-D Kolmogorov turbulence. The schematic drawing of the idea is shown in Figure 15, and an example of a set of the parameters for the two phase screens is tabulated in Table 2. In this trial, we fixed the structure exponent. One thing we have to note is that the new model fitting result is sensitive to the altitude and structure coefficient of the second screen but not to the wind velocity. The additional screen must be located at much higher than the lower phase screen, but the turbulent amplitude is about one order of magnitude smaller than the lower one. This result indicates that there exists a large scale atmospheric turbulence which can be firstly measured in ALMA interferometric observations. 

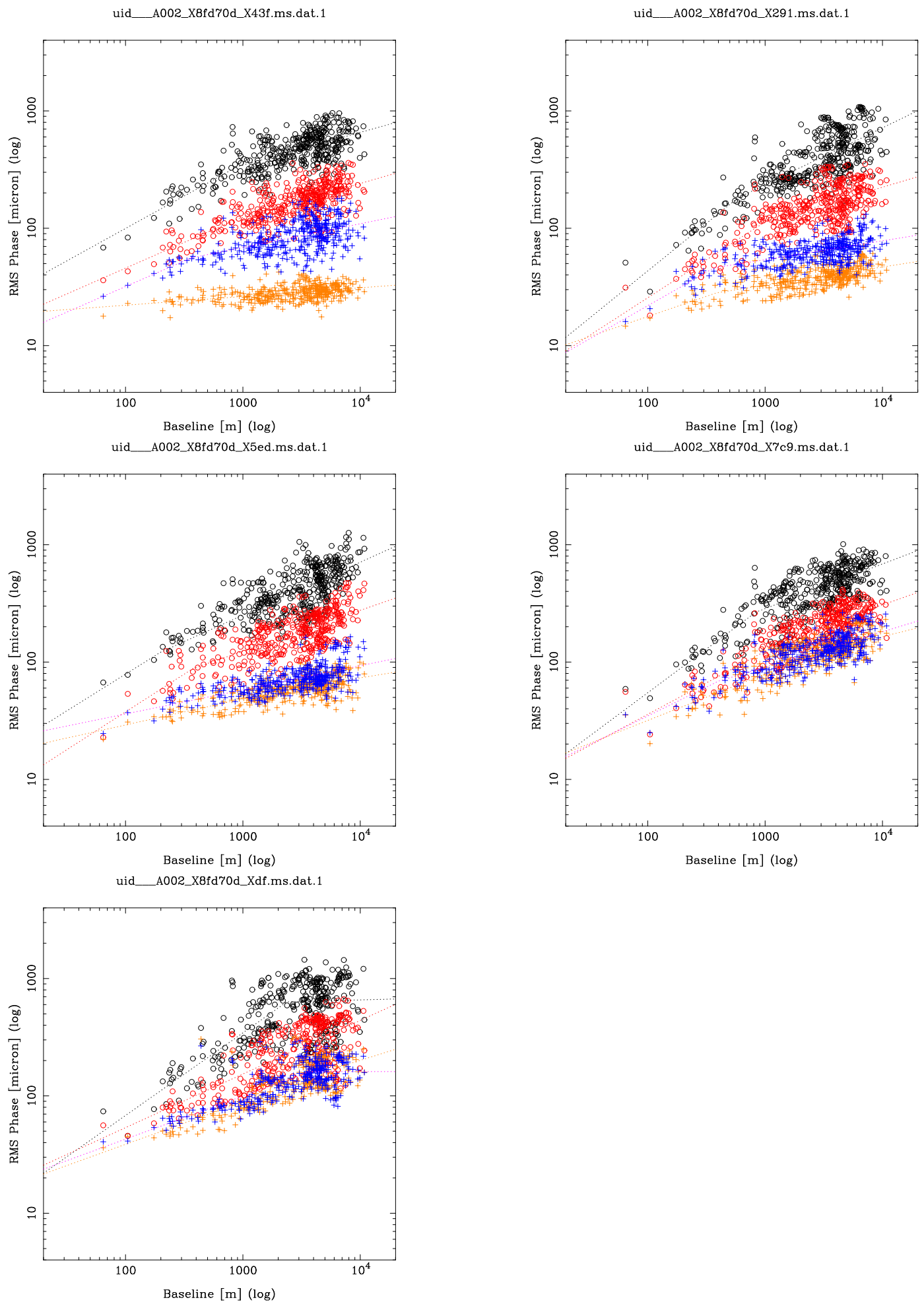

Figure 13: The same as Figure 2 but of a series of experiments of No.4 to 8 listed in Table 1. The separation angles are $0.9^{\circ}, 1.6^{\circ}, 2.6^{\circ}, 5.1^{\circ}$, and $5.5^{\circ}$ in the top left and right, middle left and right, and the bottom panels, respectively. 

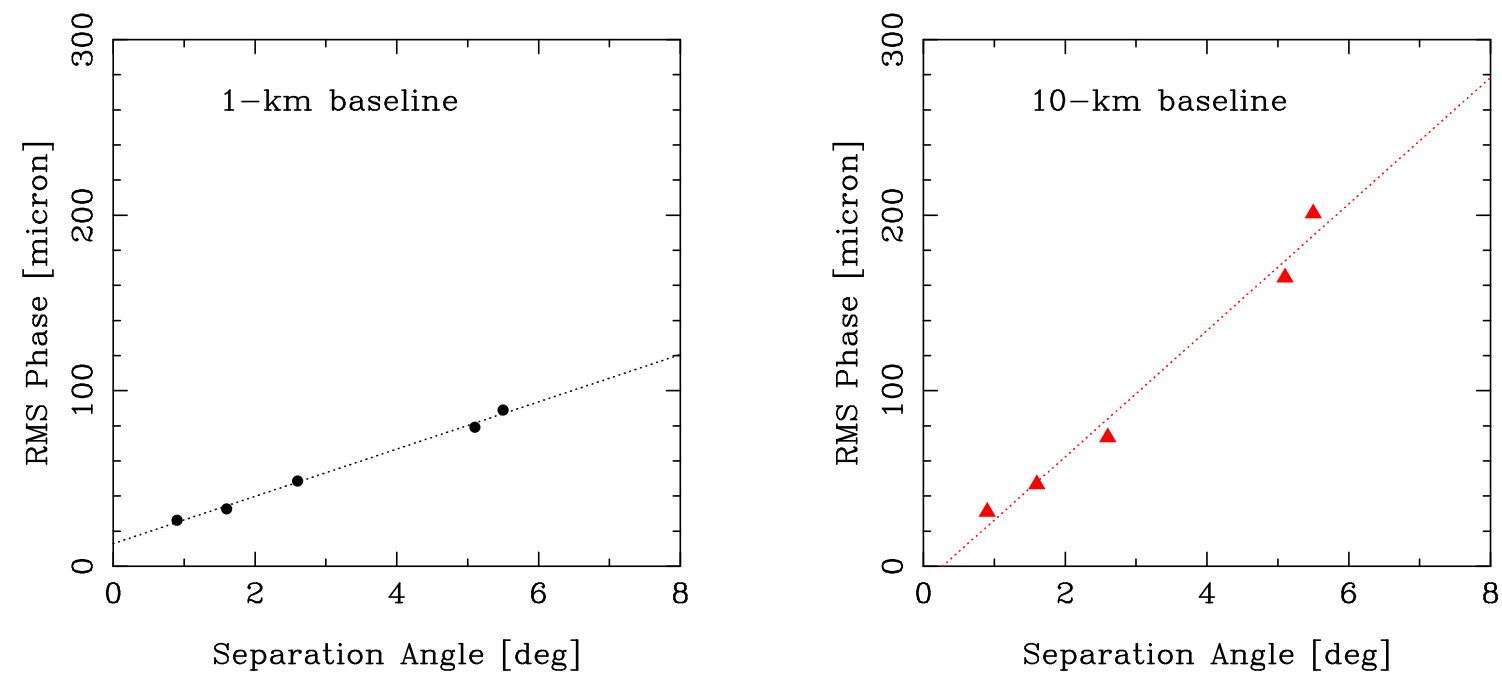

Figure 14: Rms phase of the WVR+FS-corrected interferometer phase of No.4 to 8 experiments in Table 1 as a function of separation angle. The symbols are the same as Figures 11 and 12. The red dotted lines are linear line fitting results. Left: $1 \mathrm{~km}$ baseline. Right: $10 \mathrm{~km}$ baseline.

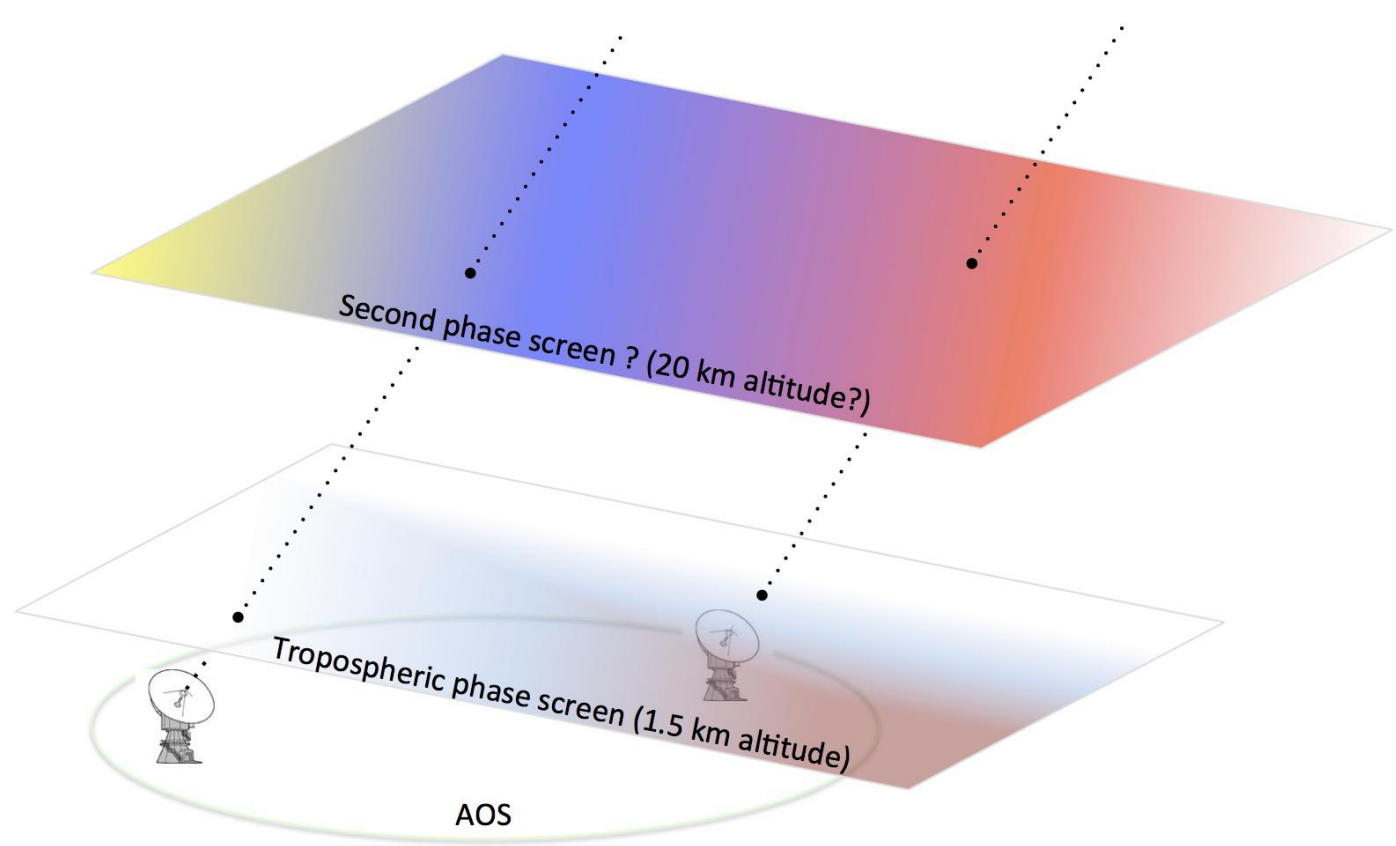

Figure 15: Schematic drawing of the atmospheric phase fluctuation model to explain the WVR+FS performance dependency on the separation angle. 
Table 2: Phase screen parameters for the model fitting of FS test experiment results (No.4 to 8).

\begin{tabular}{lc}
\hline \hline \multicolumn{1}{c}{ Tropospheric phase screen } \\
\hline Height & $1.5 \mathrm{~km}$ \\
Velocity & $15 \mathrm{~m} \mathrm{~s}^{-1}($ East to West $)$ \\
Structure coefficient $(A)$ & $1.5 \times 10^{-7} \mathrm{~m}^{-1 / 3}$ \\
Structure exponent & $5 / 3(<1 \mathrm{~km})$ \\
& $2 / 3(>1 \mathrm{~km}$ and $<8 \mathrm{~km})$ \\
& $0(>8 \mathrm{~km})$ \\
\hline \hline \multicolumn{2}{c}{ Second phase screen } \\
\hline Height & $20 \mathrm{~km}$ \\
Velocity & $100 \mathrm{~m} \mathrm{~s}^{-1}($ East to West $)$ \\
Structure coefficient $(A)$ & $6.0 \times 10^{-8} \mathrm{~m}^{-1 / 3}$ \\
Structure exponent & $5 / 3(<10 \mathrm{~km})$ \\
& $0(>10 \mathrm{~km})$ \\
\hline \hline
\end{tabular}

\section{SUMMARY}

In the ALMA Long Baseline Campaigns in 2014 and 2015, we have investigated the feasibility of WVR+FS(+B2B) phase correction scheme with the long baselines up to $16 \mathrm{~km}$. The performance is basically very promising for the advanced ALMA phase calibration especially for long baseline observations. For the separation angle smaller than $\sim 3^{\circ}$, the shorter switching cycle time can yield better performance, so that it is preferable that a bright phase calibrator within the above separation angle criterion is used with the switching cycle time of a few tens of seconds. Longer switching cycle time of 1 to $2 \mathrm{~min}$ is available where the separation angle is larger than the above because the performance is not rapidly degraded with the switching cycle time. On the other hand, it was found that the performance cannot be correctly predicted or suitably managed only with the observation parameters of the separation angle and switching cycle time.

In LBC21015, the feasibility of the WVR+FS+B2B phase correction scheme was investigated for solving the difficulty of the phase calibrator availability at high frequencies. There is no difference in the performance so far between with and without $\mathrm{B} 2 \mathrm{~B}$, so that the $\mathrm{WVR}+\mathrm{FS}+\mathrm{B} 2 \mathrm{~B}$ phase correction is also very promising as well.

In addition, we obtained some useful knowledge for the successful phase correction from our FS test experiments: (1) WVR phase correction works for $95 \%$ observations while it woks less effectively in the case that PWV is very small, (2) The FS phase referencing sometimes does not work well even for the case of the separation angle of $3^{\circ}$ maybe because of large scale blobs in the atmosphere, and (3) The atmospheric phase fluctuations occur not only in the lower troposphere, but also in the upper atmosphere with the altitude of a few tens of $\mathrm{km}$.

\section{ACKNOWLEDGMENTS}

The authors thank to all the ALMA staff in Chile to support the researches achieved in the ALMA LBCs. Y. A and S. M was adopted as the JAO ALMA expert visitors during their stays. Y. A was financially supported by the East Asia ALMA Regional Center of the National Astronomical Observatory of Japan for staying in Chile to take part in the ALMA LBCs.

\section{REFERENCES}

[1] ALMA Partnership, Brogan, C. L., Pérez, L. M., Hunter, T. R., Dent, W. R. F., Hales, A. S., Hills, R. E., Corder, S., Fomalont, E. B., and Vlahakis, C. et al., "The 2014 ALMA long baseline campaign: First results from high angular resolution observations toward the HL Tau region," ApJL 808L, 3A (2015).

[2] ALMA Partnership, Vlahakis, C., Hunter, T. R., Hodge, J. A., Pérez, L. M., Andreani, P., Brogan, C. L., Cox, P., Martin, S., and Zwaan, M. et al., "The 2014 ALMA long baseline campaign: Observations of the strongly lensed submillimeter galaxy HATLAS J090311.6+003906 at $z=3.042$," ApJL 808L, 4A (2015). 
[3] ALMA Partnership, Hunter, T. R., Kneissl, R., Moullet, A., Brogan, C. L., Fomalont, E. B., Vlahakis, C., Asaki, Y., Barkats, D., and Dent, W. R. F. et al., "The 2014 ALMA long baseline campaign: Observations of Asteroid 3 Juno at 60 kilometer resolution," ApJL 808L, 2A (2015).

[4] Asaki, Y., Matsushita, S., Morita, K.-I., and Nikolic, B., "ACA phase calibration scheme with the ALMA water vapor radiometer," in [Ground-based and Airbone Telescops IV], Stepp, L. M., Gilmozzi, R., and Hall, H. J., eds., Proc. SPIE 8444, 84443K (2012).

[5] Asaki, Y., Matsushita, S., Kawabe, R., Fomalont, E. B., Barkats, D., and Corder, S., "ALMA fast switching phase calibration on long baselines," in [Ground-based and Airbone Telescops V], Stepp, L. M., Gilmozzi, R., and Hall, H. J., eds., Proc. SPIE 9145, 91454K (2014).

[6] Matsushita, S., Asaki, Y., Kawabe, R., Fomalont, E. B., Barkats, D., and Corder, S., "Phase characteristics of the ALMA 3-km baseline data," in [Ground-based and Airbone Telescops V], Stepp, L. M., Gilmozzi, R., and Hall, H. J., eds., Proc. SPIE 9145, 91453I (2014).

[7] Matsushita, S., Asaki, Y., Fomalont, E. B., Barkats, D., Corder, S. A., Hills, R. E., Kawabe, R., Maud, L. T., Morita, K.-I., Nikolic, B., J.-Tilanus, R. P., and Vlahakis, C., "Atmospheric phase characteristics of the ALMA long baseline," in [Astronomical telescopes + instrumentation], Hall, H. J., Gilmozzi, R., and Marshall, H. K., eds., Proc. SPIE, 9906-186 (2016).

[8] Nikolic, B., Bolton, R. C., Graves, S. F., Hills, R. E., and Richer, J. S., "Phase correction for ALMA with 183 ghz water vapor radiometers," Astron. Astrophys. 552, A104 (2013).

[9] Holdaway, M. A., Carilli, C., and Laing, R., "Finding fast switching calibrators for ALMA," ALMA Memo 493 (2004).

[10] Nikolic, B., Richer, J. S., and Hills, R. E., "Simulating atmospheric phase errors, phase correction and the impact on ALMA science," ALMA Memo 582 (2008).

[11] ALMA Partnership, Fomalont, E. B., Vlahakis, C., Corder, S., Remijan, A., Barkats, D., Lucas, R., Hunter, T. R., Brogan, C. L., and Asaki, Y. et al., "The 2014 ALMA long baseline campaign: An overview," ApJL 808L, 1A (2015).

[12] Beasley, A. and Conway, J., "VLBI phase-referencing," in [Very Long Baseline Interferometry and the VLBA], ASP conference series 82, 327-343 (1995).

[13] Tatarskii, V. I., [Wave propagation in a turbulent medium], Dover, Mineola, New York (1961).

[14] Asaki, Y., Saito, M., Kawabe, R., Morita, K.-I., and Sasao, T., "Phase compensatio experiments with the paired antennas method," Radio Sci. 31, 1615-1625 (1996).

[15] Dravskikh, A. F. and Finkelstein, A. M., "Tropospheric limitations in phase and frequency coordinate measurements in astronomy," Astrophy. Space Sci. 60, 251-265 (1979).

[16] Treuhaft, R. N. and Lanyi, G. E., "The effect of the dynamic wet troposphere on radio interferometric measurements," Radio Sci. 22, 251-265 (1987).

[17] McMullin, J. P., Waters, B., Schiebel, D., Young, W., and Golap, K., "CASA architechture and applications," in [Astronomical Data Analysis Software and Systems XVI], Shaw, R. A., Hills, F., and Bell, D. J., eds., ASP Conf Ser. 376, 127-130 (2007).

[18] Nikolic, B., Graves, S. F., Bolton, R. C., and Richer, J. S., "Design and imprementation of the wvrgcal program," ALMA Memo 593 (2012).

[19] Carilli, C. L. and Holdaway, M. A., "Tropospheric phase calibration in millimeter interferometry," Radio Sci. 34, 817-840 (1999).

[20] Thompson, A. R., Moran, J. M., and Swenson, J. G. W., [Interferometry and synthesis in radio astronomy], A Wiley-Interscience Publication, John Wiley \& Sons, Inc., New York, second ed. (2001).

[21] Asaki, Y., Shibata, K. M., Kawabe, R., Roh, D.-G., Saito, M., Morita, K.-I., and Sasao, T., "Phase compensatio experiments with the paired antennas method II: Millimeter-wave fringe correction using centimeter-wave reference," Radio Sci. 33, 1297-1318 (1998).

[22] Dodson, R. and Rioja, M., "Astrometric calibration of mm-VLBI using source/frequency referenced observations," VLBA Sci. Memo 31 (2009). 
[23] Dodson, R., Rioja, M., Jung, T.-H., Sohn, B.-W., Byun, D.-Y., Cho, S.-H., Lee, S.-S., Kim, J., Kim, K.-T., and $\mathrm{Oh}, \mathrm{C}$-.S. et al., "Astrometrically registered simultaneous observations of the $22 \mathrm{GHz} \mathrm{H}_{2} \mathrm{O}$ and $43 \mathrm{GHz}$ $\mathrm{SiO}$ masers toward R Leonis Minoris using KVN and source/frequency phase referencing," $A J$ 148, 97-105 (2014).

[24] Asaki, Y., Sudou, H., Kono, Y., Doi, A., Dodson, R., Pradel, N., Mochizuki, N., Edwards, P. G., Sasao, T., and Fomalont, E. B., "Verification of the effectiveness of VSOP-2 phase referencing with a newly developed simulation tool, ARIS," PASJ 59, 397-418 (2007). 